\title{
Characteristics of wind turbine wake and particle transport in aeolian sand environment
}

\author{
Mandi Sha ${ }^{1 *}$, Xianbin Xiao ${ }^{2}, W u$ Qin $^{3}$, and Zongming Zheng ${ }^{4}$ \\ College of new energy, North China Electric Power University, 102206 Beijing, China
}

\begin{abstract}
For the wind turbine wake field in the aeolian sand environment, the multiphase flow model is used to simulate the wind sand inflow and the wake of the wind turbine is simulated and calculated in combination with the actuation line model based on the OpenFOAM-v2006. The effects of clean air inflow and wind sand inflow on the wind turbine wake field are compared, including speed loss and wake structure. Equally the particle transport in the wake of wind turbine is analyzed. The results show that the velocity of wind turbine wake recovers faster in the aeolian sand environment, the overall spatial structure is sinking, and the particles will settle in advance and distribute in strips.
\end{abstract}

Keywords: ALM, OpenFOAM, MPPICFoam, LES.

\section{Introduction}

In Northwest and North China, the wind resource rich area highly coincides with the aeolian sand area, and the influence of wake effect must be considered in the micro site selection and layout of wind turbines ${ }^{[1]}$. Deshun Li analyzed the influence of wind sand inflow on blade wear through Fluent ${ }^{[2-3]}$. Congxin Yang analyzed the wake characteristics of wind turbine under clean inflow by actuation line model simulation ${ }^{[4]}$. Due to the solid modeling method is limited by the amount of grid calculation, and cannot simulate multiple wind turbines. As a relatively accurate wake model, the actuator line model does not need solid modeling and has a small amount of calculation. At present, its application is mainly concentrated in single-phase flow system, and there is little research on the application of actuation line in multiphase flow system.

\section{Theory and model}

\subsection{Multiphase flow model(MP-PIC)}

As a particle tracking model, MP-PIC model describes the gas phase by Euler method, calculates the relative motion with the discrete phase by Lagrange method, and processes the collision between particles by calculating the pressure gradient force to reduce the amount of calculation.

\footnotetext{
*Corresponding author: smd18801096587@163.com
} 
Gas phase continuity equation:

$$
\frac{\partial}{\partial t}\left(\varepsilon_{g} \rho_{g}\right)+\nabla\left(\varepsilon_{g} \rho_{g} \mathbf{u}_{\mathbf{g}}\right)=0
$$

Gas phase momentum equation:

$$
\left\{\begin{array}{c}
\frac{\partial}{\partial t}\left(\varepsilon_{g} \rho_{g} \mathbf{u}_{\mathbf{g}}\right)+\nabla \cdot\left(\varepsilon_{g} \rho_{g} \mathbf{u}_{\mathbf{g}} \mathbf{u}_{\mathbf{g}}\right)=-\varepsilon_{g} \nabla p+\nabla \cdot \boldsymbol{\tau}_{\mathbf{g}}+\varepsilon_{g} \rho_{g} \mathbf{g}-\sum_{p=1}^{n_{T}} n_{p} \frac{V_{p}}{V_{g}} \beta_{p}\left(\mathbf{u}_{\mathbf{g}}\left(\mathbf{x}_{\mathbf{p}}\right)-\mathbf{u}_{\mathbf{p}}\right) \\
\boldsymbol{\tau}_{\mathbf{g}}=\mu_{g}\left[\nabla \mathbf{u}_{\mathbf{g}}+\left(\nabla \mathbf{u}_{\mathbf{g}}\right)^{T}\right]-\frac{2}{3} \mu_{g} \nabla \mathbf{u}_{\mathbf{g}} \mathbf{I}
\end{array}\right.
$$

Where: $\varepsilon_{g}$ is the porosity, $\rho_{g}$ is the gas phase density, $\mathbf{u}_{\mathbf{g}}$ is the gas phase velocity, $\mathbf{u}_{\mathbf{p}}$ is the particle velocity, $\mathbf{x}_{\mathbf{p}}$ is the particle displacement. $n_{T}$ is the total number of calculated parcels in the cell, $n_{p}$ is the number of real particles in the calculated parcels, $\mathbf{I}$ is the unit tensor, $\mathbf{g}$ is the gravitational acceleration, $\beta_{p}$ is the drag coefficient, $\boldsymbol{\tau}_{\mathbf{g}}$ is the corresponding air force, $V_{p}$ is the volume of particles in the cell, $V_{g}$ is the fluid volume in the cell.

Equation of motion of solid phase:

$$
\left\{\begin{array}{c}
\frac{d \mathbf{x}_{\mathbf{p}}}{d t}=\mathbf{u}_{\mathbf{p}} \\
\frac{d \mathbf{u}_{\mathbf{p}}}{d t}=-\frac{\nabla p}{\rho_{s}}-\frac{\nabla p_{s}}{\varepsilon_{s} \rho_{s}}+\mathbf{g}+\frac{\beta_{p}}{\rho_{s}}\left[\mathbf{u}_{\mathbf{g}}\left(\mathbf{x}_{\mathbf{p}}\right)-\mathbf{u}_{\mathbf{p}}\right] \\
p_{s}=\frac{p_{s}^{*} \varepsilon_{s}^{\alpha}}{\varepsilon_{s \cdot \max }-\varepsilon_{s}}
\end{array}\right.
$$

Where: $\rho_{s}$ is the solid density, $\varepsilon_{s, \max }$ is the maximum solid holdup, $p_{s}^{*}, \alpha$ is the inherent parameter of the model, generally ${ }^{[5]} 10<p_{s}^{*}<100,2<\alpha<5$.

Ergun-WenYu drag model is selected as the drag model for calculation. The drag model combines Wen Yu model and Ergun model ${ }^{[6]}$. Among them, the calculation of the drag force on particles in the flow field is as follows:

$$
\mathbf{F}_{\mathbf{p}}=\frac{3}{4} m \alpha_{p} \rho_{c} \frac{C_{D}}{d_{p}}\left(\mathbf{u}_{\mathbf{g}}-\mathbf{u}_{\mathbf{p}}\right)\left|\mathbf{u}_{\mathbf{g}}-\mathbf{u}_{\mathbf{p}}\right|
$$

\subsection{Wind turbine actuator line model (ALM)}

The actuator line model divides the blade into multiple micro elements according to BEM(blade element momentum theory). When the fluid flows through the blade, the aerodynamic force on each section is obtained by interpolation calculation according to the two-dimensional airfoil aerodynamic characteristic parameters of each blade element section, and the calculated volume force is substituted into the flow field to calculate the information of the surrounding flow field. The aerodynamic volume force is calculated as follows:

$$
\mathbf{f}_{\mathbf{a e}}=(\mathbf{L}, \mathbf{D})^{T}=\frac{1}{2} \rho \mathbf{W}^{2} c\left(C_{L} \mathbf{e}_{\mathbf{L}}, C_{D} \mathbf{e}_{\mathbf{D}}\right)^{T}
$$

Where, $\mathbf{W}$ is the local wind speed, in $m \cdot s^{-1} ; c$ is the chord length, in $m ; \rho$ is the local air density, in $\mathrm{kg} \cdot \mathrm{m}^{-3} ; C_{L}$ is the airfoil lift coefficient, $C_{D}$ is airfoil drag coefficient; $\mathbf{e}_{\mathbf{L}}, \mathbf{e}_{\mathbf{D}}$ are unit vectors in the direction of lift and drag respectively. 


\subsection{Large eddy simulation method (LES)}

In order to capture the vortex system structure in the wake, the turbulence model adopts large eddy simulation (LES). In the LES turbulence model, the Smagorinsky model overestimates the turbulent viscosity, so the solution is relatively stable and the calculation speed is fast. It is the most widely used LES model at present.

\subsection{Physical model and inflow conditions}

The simulation target in this paper is the wind turbine experiment of aeolian sand environment based on the Environmental Wind Tunnel of the Key Laboratory of Western Disaster and Environment of Lanzhou University. Due to the large calculation demand of the particle tracking model, the numerical simulation model is the model unit of wind tunnel test, and the calculation object is the reduced scale model. Based on this, the gas-solid flow characteristics of the wind turbine wake of the scale model are simulated and analyzed.

The calculation area ranges from $2 \mathrm{D}$ in front of the wind turbine to $20 \mathrm{D}$ behind the wind turbine, with a size of $1.3 \mathrm{~m} \times 1.4 \mathrm{~m} \times 10.8 \mathrm{~m}(W \times H \times L)$. The grid densification area ranges from $0.5 \mathrm{D}$ in front of the wind turbine to $15 \mathrm{D}$ behind the wind turbine, with a size of $0.6 \mathrm{~m} \times 0.6 \mathrm{~m} \times 6.82 \mathrm{~m}(W \times H \times L)$ and adopt SnappyHexMesh for grid encryption. The total number of grids is 5 million. Fig. 1 shows the grid division used in the calculation, where $\mathrm{A}$ is the layout position of the wind turbine, $\mathrm{B}$ is the grid densification area, and $\mathrm{C}$ is the fluid domain of the calculation.

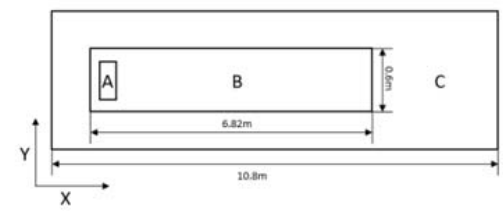

Fig. 1. Diagrammatic sketch of meshing.
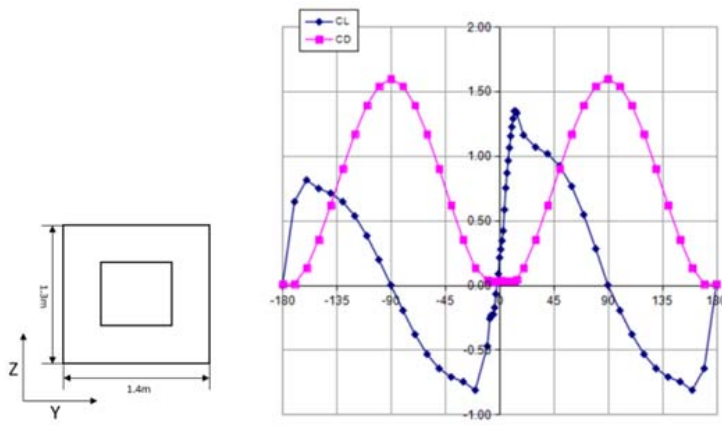

Fig. 2. SG5060 Airfoil lift and drag coefficient.

The boundary settings and wind turbine parameter settings are shown in Table 1.

Table 1. Basic parameters.

\begin{tabular}{lcc}
\hline Parameter & Numerical value & Unit \\
\hline Incoming flow velocity & 8 & $\mathrm{~m} / \mathrm{s}$ \\
Tip speed ratio & 4 & $/$ \\
Number of blades & 3 & $/$ \\
Airfoil & $\mathrm{SG} 6050$ & $/$ \\
Diameter of wind turbine & 0.44 & $\mathrm{~m}$ \\
Hub height & 0.675 & $\mathrm{um}$ \\
Particle size & 70 & $/$ \\
Number of parcels per second & 17400321 & $/$ \\
Number of particles per parcel & 1000 & $\mathrm{~kg} / \mathrm{m}^{3}$ \\
Particle density & 2560 & \\
\hline
\end{tabular}

According to the blade element momentum theory, the aerodynamic design of wind turbine blade is carried out. Different Airfoils are selected for different spanwise lengths, and the lift coefficient and drag coefficient are interpolated within the angle of attack range 
of $0^{\circ}-180^{\circ}$. The results are shown in the Fig. 2.

\section{Results and discussion}

This paper simulates and analyzes the gas-solid flow characteristics in the test section, compares the wind turbine wake flow field of clean air and the wind turbine wake flow field under the incoming sand, and studies the influence of particles on the temporal and spatial evolution of wake and the influence of wind turbine wake on particle transport.

\subsection{Structure and velocity recovery of wind turbine wake}

Under the inflow condition of $8 \mathrm{~m} / \mathrm{s}$ under the rated working condition of the wind turbine, the wake velocity distribution curve of the wind turbine presents an M-shaped distribution and recovers with the distance. Compare the velocity distribution of wind turbine wake field under clean air and sand, as shown in Figure 3 and select the position 1D, 5D, 10D and $15 \mathrm{D}$ behind the wind turbine to make the velocity distribution of $\mathrm{XY}$ section and $\mathrm{XZ}$ section, as shown in Fig. 4.

(a)

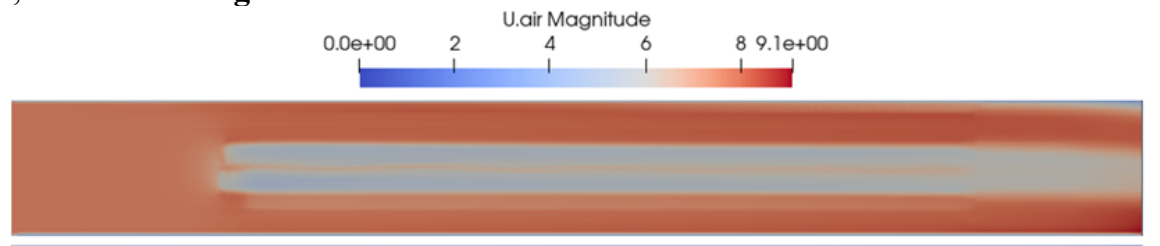

(b)

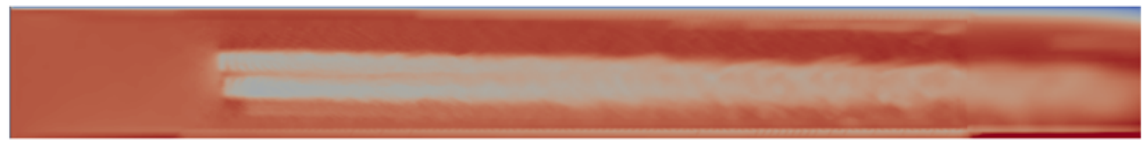

Fig. 3. Velocity cloud diagram of wind turbine wake field (a) clean air inflow (b) wind sand inflow.

For the wind sand flow, the settlement effect caused by the gravity of the particles will be transmitted to the wind turbine wake through the drag force between the gas phase and the particle phase, resulting in the downward trend of the wind turbine wake in space. Fig. 4 (b) shows the flow field velocity cloud diagram of XZ section.

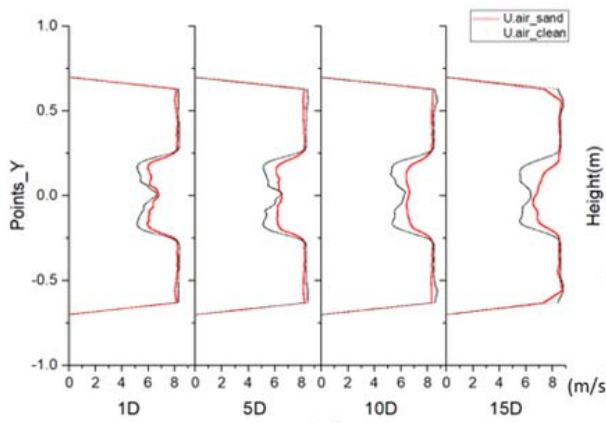

(a)

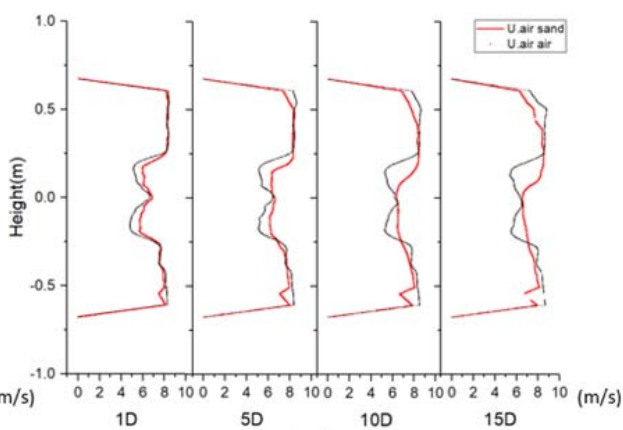

(b)

(a) $\mathrm{XY}$ section (b) $\mathrm{XZ}$ section

Fig. 4. Comparison of wake velocity under the condition of clean air inflow and wind sand inflow.

The minimum value of the velocity profile of each section in Fig. 4 are selected as the characteristic quantity for comparative analysis, and the results are shown in Table 2. Due to the influence of particle movement, the air flow is more disordered and the wake of the 
wind turbine will recover relatively quickly.

Table 2. Comparison of wind turbine wake loss under different incoming flows.

\begin{tabular}{lllll}
\hline Parameter & 1D & 5D & 10D & $15 \mathrm{D}$ \\
\hline Clean air & 5.11258 & 5.15867 & 5.34404 & 5.54289 \\
Velocity loss & 0.36092 & 0.35516 & 0.33199 & 0.30713 \\
Wind sand flow & 5.89507 & 6.11965 & 6.39141 & 6.51209 \\
Velocity loss & 0.26311 & 0.23504 & 0.20107 & 0.18598 \\
\hline
\end{tabular}

\subsection{Influence of wind turbine wake on particle transport}

When the air flows through the plane of the wind turbine rotor, the wind machine absorbs energy from the flowing air, resulting in the loss of air energy and the decrease of speed. At this time, due to the drag force of the gas-phase fluid, the particles will also decelerate accordingly. Due to the constant existence of gravity and the weakening of the upward velocity of the square of water, the particles will settle in advance. Due to the wind turbine wake structure is three periodically changing spirals, the particles will show a banded distribution during deposition due to the influence of the wake.

The number of particles from the surface to $0.44 \mathrm{~m}$ above the ground is analyzed. Fig. 5 shows that there will be a peak value of the number of particles every $1 \mathrm{~m}$ along the incoming flow direction, which is similar to the periodic change of the wind turbine wake in space. Compared with the partial velocity of the flow field in the Y direction at this position, shown in Fig. 6, the changing value is similar to the distribution of the number of particles, It shows that the wind turbine wake can promote the strip deposition of particles.

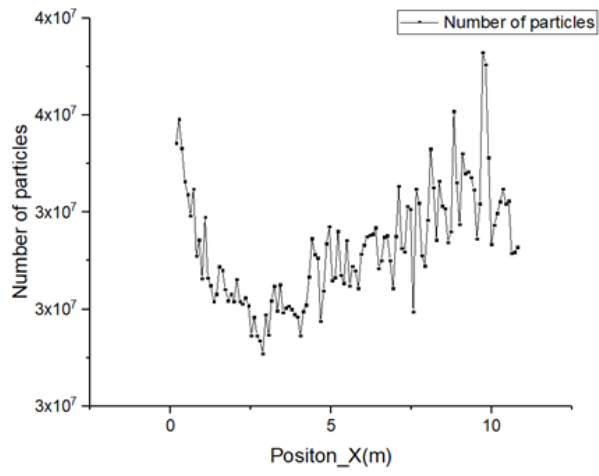

Fig. 5. Number of particles from ground to $0.44 \mathrm{~m}$ above.

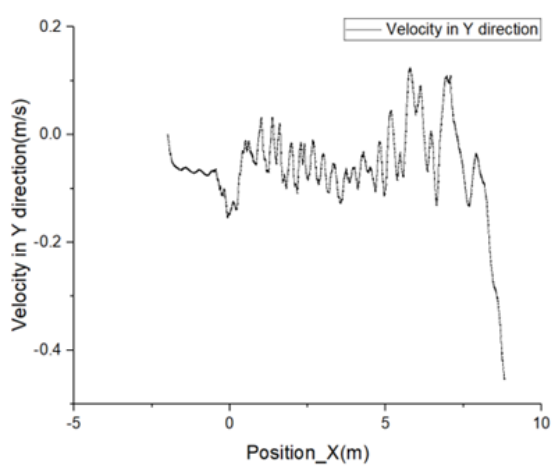

Fig. 6. Velocity of Y direction.

\section{Conclusion}

In this paper, multiphase flow model and actuation line model are coupled based on OpenFOAM-v2006 in order to simulate the temporal and spatial changes of wind turbine wake under clean air and wind sand inflow. The loss and morphological structure of wind turbine wake are analyzed, and the influence of wind turbine wake on particle transport is concerned.

1) Because of the movement of particles in the flow field, the wind turbine wake is more disturbed, the turbulent kinetic energy increases, and the wake recovery increases.

2) Due to its own gravity, particles are transmitted to the air through the drag force between the fluid and solid phases, resulting in the sinking of the wind turbine wake field in the spatial structure. 
3) Under the periodic rotation of wind turbine blades, the change of wake has periodic characteristics. Therefore, the particles will slow down due to the influence of wind turbine wake during the settlement process, resulting in early deposition and strip distribution.

As a result of the simple grid required by the actuation line model, the wake simulation of multiple units can be expanded in the future to study the temporal and spatial evolution of the wake structure of the wind farm in the aeolian sand environment, so as to optimize the layout and improve the power generation efficiency.

\section{References}

1. Energy - Wind Energy; Studies from Technical University Further Understanding of Wind Energy (Analytical model for the power-yaw sensitivity of wind turbines operating in full wake)[J]. Energy Weekly News (2020)

2. Li Deshun, Chen Xia, Li Yinran, Guo xingduo, Wang Yae Study on the evolution process of wind sand erosion wear of wind wing [J] Gansu Journal of science 32, 05 (2020),32(05):63-68+87.DOI:10.16468/j.cnki.issn1004-0366.2020.05.013.

3. Li rennian, Zhao Zhenxi, Li Deshun, Li Yinran, Chen Xia, Yu Jiaxin Effect of wind sand on the flow around wind wing and its aerodynamic performance $[\mathrm{J}]$ Journal of agricultural engineering 34, 14(2018),34(14):205-211+303.

4. Yang Congxin, Zhang Yaguang, Zhang xuyao, He pan Study on wake characteristics of wind turbine based on large eddy simulation $[\mathrm{J}]$ Mechanical design and manufacturing (2021)

5. Auzerais F M, Jackson R, Russel W B. The resolution of shocks and the effects of compressible sediments in transient settling [J]. J. Fluid Mech (2006)

6. GIDASPOW D. Multiphase flow and fluidization: continuum and kinetic theory descriptions [M]. Academic press 42 (1994) 